\title{
Effect of the magnetocrystalline anisotropy on the magnetic behavior of ring elements
}

\author{
C. A. F. Vaz, M. Kläui, ${ }^{a}$ and J. A. C. Bland ${ }^{\text {b) }}$ \\ Cavendish Laboratory, University of Cambridge, Cambridge CB3 OHE, United Kingdom \\ L. J. Heyderman \\ Laboratory for Micro- and Nanotechnology, Paul Scherrer Institut, Villigen PSI, Switzerland \\ F. Nolting \\ Swiss Light Source, Paul Scherrer Institut, Villigen PSI, Switzerland
}

(Presented on 6 January 2004)

\begin{abstract}
We systematically compare the magnetic properties of epitaxial and polycrystalline Co disks and rings with varying width $(110-730 \mathrm{~nm})$ for a fixed Co thickness and outer diameter $(16 \mathrm{~nm}$ and $\sim 1.6 \mu \mathrm{m}$, respectively) by magneto-optical Kerr microscopy and photoemission electron microscopy. The magnetic hysteresis loops of the epitaxial (fcc Co) samples exhibit much larger switching fields and broader switching field distributions than the polycrystalline structures. This behavior is attributed to the different origin of the pinning fields in, respectively, the epitaxial and polycrystalline structures. Our results show also that for epitaxial rings, the magnetic global easy axis is along the magnetocrystalline hard axis. (C) 2004 American Institute of Physics.
\end{abstract}

[DOI: $10.1063 / 1.1667449$ ]

The study of the magnetic properties of lithographically patterned elements is now a key area of research in physics, not only due to its importance in technological applications ${ }^{1}$ but also for the insights it provides into nanoscale magnetism. Fundamental issues are, for example, the effect of the magnetocrystalline anisotropy and dipolar interactions on the magnetic switching and equilibrium states, ${ }^{2-8}$ and transport properties. ${ }^{9-13}$ A critical parameter in small elements is the physical shape, since it determines to a large extent both the static (equilibrium) and dynamic (switching) magnetic configurations that are accessible to the system. This is due to the long range dipolar interactions, which act in such a way as to reduce the magnetostatic self-energy by favoring fluxclosure configurations. The effect of the magnetocrystalline anisotropy is usually that of introducing local domains pointing along the easy direction axis. ${ }^{5}$ One system where this interplay between the different energy terms is striking is that of the disk with cubic magnetic anisotropy, where the equilibrium magnetic configuration corresponds to a hybrid between a vortex state and a quadrant (Landau-Lifschitz) state. ${ }^{5}$ Another example occurs in rings with cubic anisotropy (as in fcc Co rings), which is responsible for the creation of small local magnetic domains. ${ }^{6}$ Also, the kind of walls present in rings in the "onion" state (where the magnetization of the two halves of the ring point in the same direction ${ }^{14}$ depends on the energy competition between the dipolar interactions and the exchange energy, leading either to transverse walls for narrow rings or vortex walls for wide rings. ${ }^{6,15}$ These conflicting energy contributions lead to a rich

\footnotetext{
${ }^{a)}$ Present address: Fachbereich Physik, Universität Konstanz, Universitätsstr. 10, D-78457 Konstanz, Germany.

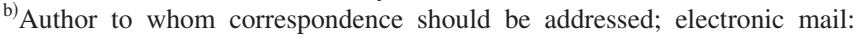
jacb1@phy.cam.ac.uk
}

magnetic behavior that is a function of the geometrical parameters of the ring and of the magnetic material employed and which could be harnessed for particular applications. ${ }^{16,11,17}$ However, to date, the role of the magnetic anisotropy has not been so extensively investigated. In this study we consider the presence of a magnetocrystalline anisotropy on the magnetic behavior of ring elements by a systematic study of the $M-H$ characteristics and also by the imaging of the magnetic states at remanence.

The samples consist of epitaxial fcc Co or polycrystalline hcp Co films which were deposited on a poly(methylmethacrylate) patterned $\mathrm{Si}(001)$ substrate followed by a liftoff step. ${ }^{18,19}$ The patterned elements consist of arrays of rings with varying inner diameter, ranging from narrow rings to disks, with ring width $w=110,260,350,530,730 \mathrm{~nm}$, and outer diameter set to $\sim 1.6 \mu \mathrm{m}$. The spacing between the rings is such that the interaction between the rings is negligible (this is the case when the distance separating the elements is larger than the physical dimension of the element ${ }^{20}$ ). The epitaxial fcc Co film was obtained by deposition of a thick $\mathrm{Cu}$ buffer layer onto the HF etched $\mathrm{Si}$ substrate, while the polycrystalline Co samples (with no overall magnetic anisotropy) were obtained by deposition of the Co film directly onto the oxidized $\mathrm{Si}$ surface. ${ }^{21}$ The fabrication of the epitaxial Co rings poses some problems due to the thick $\mathrm{Cu}$ buffer layer, in that for very thick metal layers it becomes difficult to do the lift-off process successfully. This was the case for one of the epitaxial samples, $0.5 \mathrm{~nm} \mathrm{Al} / 1.3 \mathrm{~nm}$ $\mathrm{Cu} / 29 \mathrm{~nm} \mathrm{Co} / 75 \mathrm{~nm} \mathrm{Cu} / \mathrm{Si}(001)$, where for a large percentage of the rings the central core could not be removed. This gives rise to additional jumps in the $M-H$ loop which are not due to the rings. For the thinner sample, $0.5 \mathrm{~nm} \mathrm{Au} / 16 \mathrm{~nm}$ $\mathrm{Co} / 37 \mathrm{~nm} \mathrm{Cu} / \mathrm{Si}(001)$, the lift-off worked well. This limitation in the metal thickness achievable is common to all pat- 

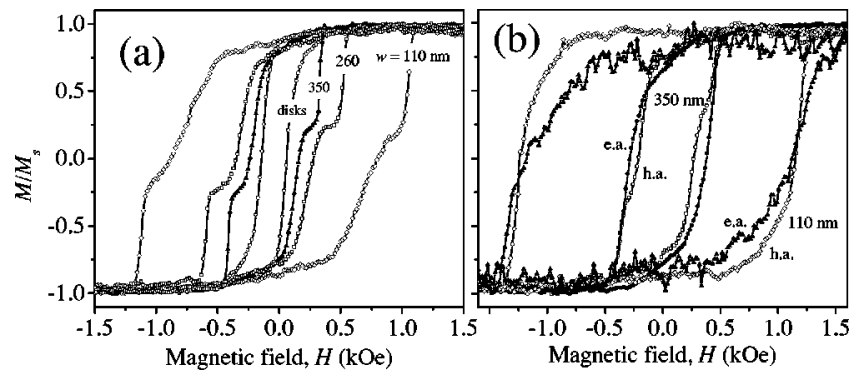

FIG. 1. MOKE measurements on (a) polycrystalline Co rings $(w=110,260$, 350 and disks) and (b) for the 350 and $110 \mathrm{~nm}$ width epitaxial fcc rings with the field applied along the magnetic easy (e.a.) and hard axis (h.a.). In both samples, the switching field increases with decreasing ring width and for the epitaxial rings (b), the switching field is larger along the easy axis direction.

terning techniques, and these two examples point towards the type of difficulties that one encounters when working with these systems, although it represents by no means the limit of what may be achievable. The hysteresis loops were measured by magneto-optic Kerr effect (MOKE) magnetometry on large arrays of identical rings while the magnetic imaging was performed on smaller arrays, placed close together, convenient for the photoemission electron microscopy (PEEM) technique, ${ }^{22,23}$ conducted at the SIM beamline at the Swiss Light Source (Switzerland).

We shall concentrate here on the comparison between the magnetic properties of the $16 \mathrm{~nm}$ thickness fcc Co with the $16 \mathrm{~nm}$ polycrystalline Co rings. The $M-H$ loops for either sample show identical switching behavior as a function of the ring width: for all rings a double switching process is observed (corresponding to the switching between the onion and vortex states), and the field at which these transitions occur increases with decreasing ring width (Fig. 1). For the polycrystalline sample this has been reported before. ${ }^{4}$ The main difference between the two samples resides in the much larger switching fields for the case of the epitaxial sample, which is attributed to the stronger pinning sites for the magnetization, due to the internal stress of the Co film at the $\mathrm{Co} / \mathrm{Cu}$ interface. ${ }^{21}$ For the $110 \mathrm{~nm}$ width fcc Co rings, the switching fields are very large, and they seem to coalesce into a single switching field, in contrast to the polycrystalline rings, where the double switching is still clearly distinguishable. For the epitaxial rings, the switching field is much broader along the magnetocrystalline easy axis than when the magnetic field is applied along the hard axis. This behavior has, in fact, been suggested before by the results of micromagnetic simulations on rings with cubic anisotropy, where it was shown that for these ring structures the global magnetic easy direction is, in fact, along the magnetocrystalline hard axis. ${ }^{24}$ This is a result of the extra energy introduced by the magnetocrystalline anisotropy when the onion state is saturated along the magnetocrystalline easy axis, since in this case the magnetization along each quarter of the ring points along magnetic hard axes. This makes the onion state more unstable, and therefore easier to switch into the vortex state when a reverse field is applied.

The MOKE measurements are the result of a statistical average over many individual loops (this is necessary due to the small magnetic signal from the ring arrays). This means
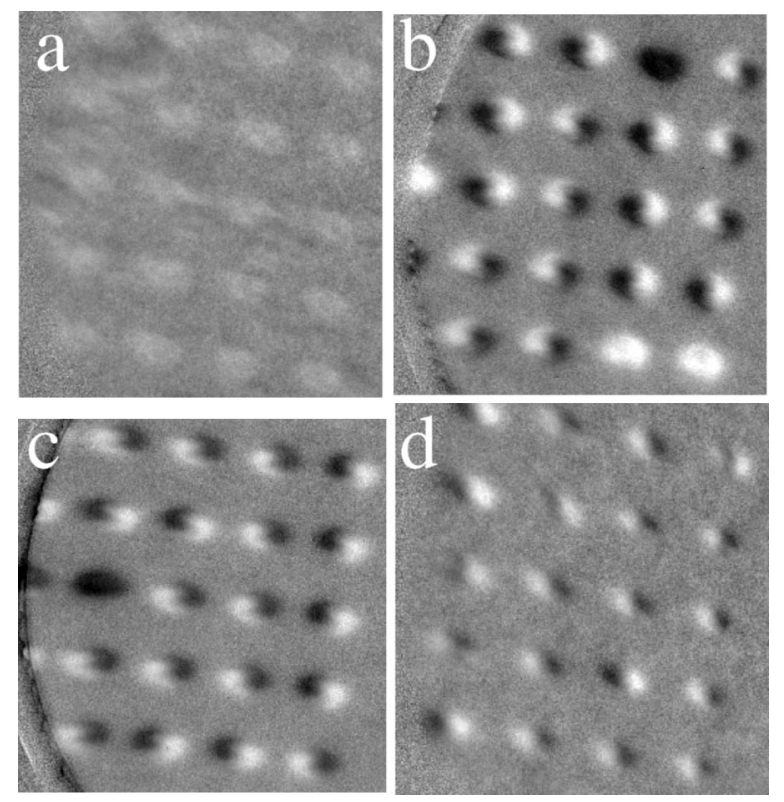

FIG. 2. PEEM images of the epitaxial $16 \mathrm{~nm}$ thickness Co structures: (a) $w=110 \mathrm{~nm}$, (b) $w=350 \mathrm{~nm}$, (c) $w=530 \mathrm{~nm}$ and (d) disks. A strong magnetic field (4 kOe) was applied first in the vertical (upward) direction, followed by a reverse field of 500 Oe (downward direction). The magnetic contrast is along the direction of the applied magnetic field.

that, by necessity, many of the individual statistical events that correspond to the switching of each individual element are washed out in the final MOKE loop. In order to follow more closely the individual switching behavior of the ring elements, we imaged the magnetic states of the rings after a given field cycle. The PEEM images for the epitaxial Co sample were performed after the sample was saturated and had been subjected to a magnetic field of 500 Oe in the opposite direction (Fig. 2). This field corresponds roughly to the switching field of the $350 \mathrm{~nm}$ width rings. We see that most of these rings switch to the vortex state, but two remain in the previous onion state while one has already switched to the opposite onion state, Fig. 2(b). For the narrower rings, this field is still enough to switch some of the rings to the vortex state for the 260-nm-wide rings (not shown), while the narrower rings remain all in the onion state. Most of the wider rings switch to the vortex state (which is energetically the most favorable) although some switch to the reverse onion state (in particular, the disks remain all in the vortex state).

For the polycrystalline elements, we imaged the magnetic states at remanence after saturation (Fig. 3). This gives an insight into the stability of the magnetic states at remanence. We see here that the narrower rings remain in the onion state after saturation, while some of the 530-nm-wide rings switch to the onion state; this percentage increases for the wider rings, where, in fact, some fall into a multidomain state. Surprisingly, many of the disks seem to remain in a single domain state, while it is known that their ground state is the vortex state. This, however, is in agreement with the large hysteresis in the corresponding $M-H$ loop. (This behavior is not significantly influenced by the thickness of the Co layer, in the range from 10 to $20 \mathrm{~nm}$.) This highly un- 

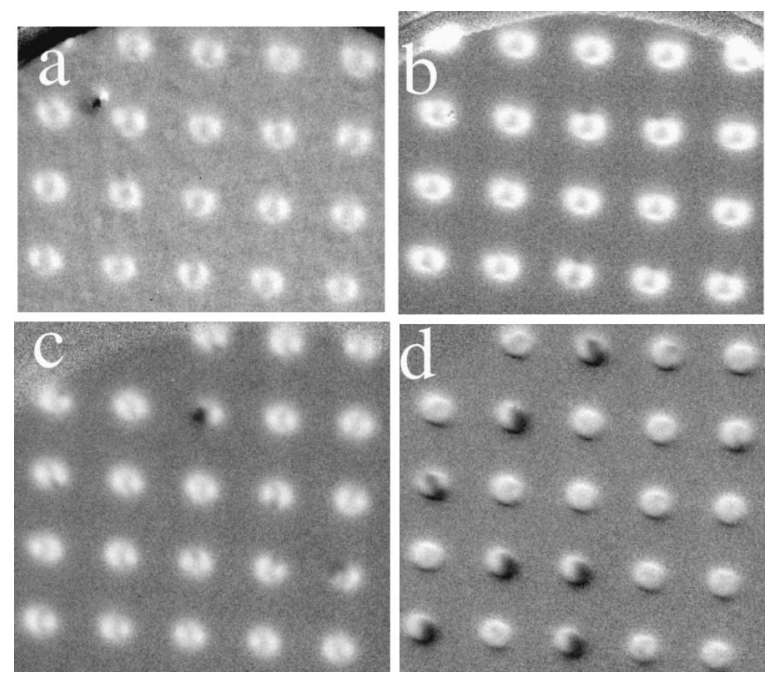

FIG. 3. PEEM images of the epitaxial $16 \mathrm{~nm}$ thickness Co structures: (a) $w=220 \mathrm{~nm}$, (b) $w=350 \mathrm{~nm}$, (c) $w=530 \mathrm{~nm}$ and (d) disks. The structures were saturated along the vertical (upward) direction (the magnetic contrast is along the same direction).

stable energy state is kept in check by the large nucleation field necessary for the creation of a vortex core at the periphery of the disk (micromagnetic simulations show that in disks the vortex state is attained by the nucleation and propagation of one or two vortex cores at the disks' positions where the stray field is maximum ${ }^{5}$ ).

In conclusion, we have presented a comparative study of the magnetic switching behavior and of the remanence magnetic states for epitaxial and polycrystalline Co ring elements. The epitaxial ring elements display larger switching fields with a broader distribution range than the polycrystalline rings. This is not attributed to the effect of the additional magnetocrystalline anisotropy (which influences instead the details of the domain configurations and the difference in switching fields between the easy and hard axis directions) but rather to the stronger pinning in the epitaxial Co films (due to internal stress at the $\mathrm{Co} / \mathrm{Cu}$ interface). Magnetic imaging of the remanent states indicates that the stability of the onion state starts to deteriorate for large ring widths (above $530 \mathrm{~nm}$ for a $16 \mathrm{~nm}$ polycrystalline Co film). We also find that many of the disks remain in a single domain state after saturation, which we suggest is a consequence of the large vortex core nucleation fields necessary for the beginning of the relaxation process towards the vortex state.
This work was supported by the EPSRC (U.K.), the E.C. MASSDOTS (EP32464), the CMI Magnetoelectronic Devices Project, and the Gottlieb Daimler-and Karl BenzFoundation (M.K.). Part of this work was performed at the SLS, Paul Scherrer Institute, Villigen, Switzerland.

${ }^{1}$ J.-G. Zhu, Y. Zheng, and G. A. Prinz, J. Appl. Phys. 87, 6668 (2000).

${ }^{2}$ R. P. Cowburn, D. K. Koltsov, A. O. Adeyeye, M. E. Welland, and D. M. Tricker, Phys. Rev. Lett. 83, 1042 (1999).

${ }^{3}$ G. Gubbiotti, L. Albani, G. Carlotti, M. D. Crescenzi, E. D. Fabrizio, A. Gerardino, O. Donzelli, F. Nizzoli, H. Koo, and R. D. Gomez, J. Appl. Phys. 87, 5633 (2000).

${ }^{4}$ Y. G. Yoo, M. Kläui, C. A. F. Vaz, L. Heyderman, and J. A. C. Bland, Appl. Phys. Lett. 82, 2470 (2003).

${ }^{5}$ C. A. F. Vaz, L. Lopez-Diaz, M. Kläui, J. A. C. Bland, T. L. Monchesky, J. Unguris, and Z. Cui, Phys. Rev. B 67, 140405(R) (2003).

${ }^{6}$ M. Kläui, C. A. F. Vaz, J. A. C. Bland, T. L. Monchesky, J. Unguris, E. Bauer, S. Cherifi, S. Heun, A. Locatelli, and L. J. Heyderman, Phys. Rev. B 68, 134426 (2003).

${ }^{7}$ F. J. Castaño, C. A. Ross, C. Frandsen, A. Eilez, D. Gil, H. I. Smith, M. Redjdal, and F. B. Humphrey, Phys. Rev. B 67, 184425 (2003).

${ }^{8}$ X. Zhu, P. Grütter, V. Metlushko, Y. Hao, F. J. Castaño, C. A. Ross, B. Ilic, and H. I. Smith, J. Appl. Phys. 93, 8540 (2003).

${ }^{9}$ T. Taniyama, I. Nakatani, H. Yanagihara, and E. Kita, J. Magn. Magn. Mater. 196, 197, 77 (1999).

${ }^{10}$ U. Ebels, A. Radulescu, Y. Henry, L. Piraux, and K. Ounadjela, Phys. Rev. Lett. 84, 983 (2000)

${ }^{11}$ M. Kläui, C. A. F. Vaz, J. A. C. Bland, W. Wernsdorfer, G. Faini, E. Cambril, and L. J. Heyderman, Appl. Phys. Lett. 83, 105 (2003).

${ }^{12}$ M. Kläui, C. A. F. Vaz, J. Rothman, J. A. C. Bland, W. Wernsdorfer, G. Faini, and E. Cambril, Phys. Rev. Lett. 90, 097202 (2003).

${ }^{13}$ C. A. F. Vaz, E. Blackburn, M. Kläui, J. A. C. Bland, L. Gan, J. W. F. Egelhoff, G. Faini, E. Cambril, and W. Wernsdorfer, J. Appl. Phys. 93, 8104 (2003).

${ }^{14}$ J. Rothman, M. Kläui, L. Lopez-Diaz, C. A. F. Vaz, A. Bleloch, J. A. C. Bland, Z. Cui, and R. Speaks, Phys. Rev. Lett. 86, 1098 (2001).

${ }^{15}$ R. D. McMichael, J. Eicke, M. J. Donahue, and D. G. Porter, J. Appl. Phys. 87, 7058 (2000).

${ }^{16}$ M. M. Miller, G. A. Prinz, S.-F. Cheng, and S. Bounnak, Appl. Phys. Lett. 81, 2211 (2002).

${ }^{17}$ N. Dao and S. L. Whittenburg, IEEE Trans. Magn. 39, 2525 (2003).

${ }^{18}$ L. J. Heyderman, M. Kläui, J. Rothman, C. A. F. Vaz, and J. A. C. Bland, J. Appl. Phys. 93, 7349 (2003).

${ }^{19}$ L. J. Heyderman, C. David, M. Kläui, C. A. F. Vaz, and J. A. C. Bland, J. Appl. Phys. 93, 10011 (2003).

${ }^{20}$ A. O. Adeyeye, J. A. C. Bland, C. Daboo, and D. G. Hasko, Phys. Rev. B 56, 3265 (1997).

${ }^{21}$ M. Kläui, C. A. F. Vaz, L. Lopez-Diaz, and J. A. C. Bland, J. Phys.: Condens. Matter 15, R985 (2003).

${ }^{22}$ A. Locatelli, K. S. Cherifi, S. Heun, M. Marsi, K. Ono, A. Pavlovska, and E. Bauer, Surf. Rev. Lett. 9, 171 (2002).

${ }^{23}$ A. Scholl, H. Ohldag, F. Nolting, J. Stöhr, and H. A. Padmore, Rev. Sci. Instrum. 73, 1362 (2002).

${ }^{24}$ L. Lopez-Diaz, J. Rothman, M. Kläui, and J. A. C. Bland, J. Appl. Phys. 89, 7579 (2001). 